\title{
Insignias digitales policiales: estudio exploratorio en la Policía Nacional de Colombia
}

\section{Police digital badges: exploratory study within National Police of Colombia Insígnias digital policiais: estudo exploratório na Polícia Nacional da Colômbia}

\author{
Jonatan Moreno Daza ${ }^{\mathrm{a}^{*}} \mid$ Catalina Cárdenas Quijano ${ }^{\mathrm{b}} \mid$ César Mauricio Rodríguez Zárate ${ }^{\mathrm{c}}$ \\ | Álvaro Yadir Castiblanco Murcia ${ }^{\mathrm{d}}$ \\ a https://orcid.org/0000-0001-6903-4047 Policía Nacional de Colombia, Bogotá, Colombia \\ ${ }^{\mathrm{b}}$ https://orcid.org/0000-0003-1886-693X Policía Nacional de Colombia, Bogotá, Colombia \\ c https://orcid.org/0000-0001-7293-2075 Policía Nacional de Colombia, Bogotá, Colombia \\ d https://orcid.org/0000-0002-7546-3291 Policía Nacional de Colombia, Bogotá, Colombia
}

- Fecha de recepción: 2021-02-07

- Fecha concepto de evaluación: 2021-04-17

- Fecha de aprobación: 2021-04-30

https://doi.org/10.22335/rlct.v13i2.1397
Para citar este artículo / To reference this article / Para citar este artigo: Moreno, J., Cárdenas, C., Rodríguez, C. M., \& Castiblanco, A. Y. (2021). Insignias digitales policiales: estudio exploratorio en la Policía Nacional de Colombia. Revista Logos Ciencia \& Tecnología,

13(2), 56-71. https://doi.org/10.22335/rlct.v13i2.1397

\section{RESUMEN}

El ritmo acelerado con el que avanza la tecnología a nivel mundial trae consigo nuevas formas de interacción humana en entornos digitales. Esta manera virtual de comunicarse e intercambiar información ha permitido el nacimiento de las insignias digitales para identificar y reconocer un logro, una destreza, una habilidad, una competencia u otro avance propio de dicha relación. El presente estudio explora las condiciones de viabilidad para implementar un sistema de insignias digitales policiales como herramienta para identificar, motivar, promover, retroalimentar y reconocer las competencias y habilidades de sus integrantes. Mediante un abordaje cualitativo, se establecen las categorías y subcategorías emergentes, así como los principales aspectos necesarios para su diseño e implementación. Teniendo en cuenta los resultados obtenidos, se concluye que un sistema de insignias digitales policiales (SIDPo) representa una oportunidad institucional para innovar, validar y reconocer las competencias y habilidades del profesional de policía en ecosistemas digitales, al facilitar la interacción digital con la comunidad y el Estado en tiempo real, especialmente en aquellos eventos en que los logros institucionales son desconocidos por la rigidez de las comunicaciones oficiales.

Palabras clave: ecosistema digital, innovación, insignia, policía, sistema

\footnotetext{
* Autor para correspondencia. Correo electrónico: jonatan.moreno@correo.policia.gov.co
} 


\begin{abstract}
The accelerated rhythm with which technology advances globally brings with it new ways of human interaction in digital environments. This digital way of communicating and exchanging information has allowed the birth of digital badges in order to identify and recognize an achievement, a skill, a competence, abilities or another matter specific to that relationship. This study explores the feasibility conditions to implement a police digital badge system as a tool to identify, motivate, promote, provide feedback and recognize the competencies, skills and abilities of its members. Using a qualitative approach, this study establishes the emerging categories and subcategories and the main aspects necessary for their design and implementation. Taking into account the results obtained, it is concluded that a system of police digital badges (SIDPo) represents an institutional opportunity to innovate, validate and recognize the competencies, skills and abilities of the police professional in digital ecosystems, facilitating digital interaction with the community and the Colombian state in real time, especially in those events in which institutional achievements are unknown due to the rigidity of official communications.
\end{abstract}

Keywords: badge, digital environment, innovation, police, system

\title{
RESUMO
}

O ritmo acelerado com que a tecnologia avança globalmente traz consigo novas formas de interação humana em ambientes digitais. Essa forma digital de comunicação e troca de informações permitiu o nascimento de insígnias digitais para identificar e reconhecer uma conquista, uma habilidade, uma expertise, uma competência ou outro avanço desse relacionamento. Este estudo explora as condições de viabilidade de implementação de um sistema de insígnias digitais policiais como ferramenta para identificar, motivar, promover, dar feedback e reconhecer as competências e habilidades dos seus membros. Por meio de uma abordagem qualitativa, são estabelecidas categorias e subcategorias emergentes, bem como os principais aspectos necessários para sua concepção e implementação. Levando em consideração os resultados obtidos, conclui-se que um sistema de insígnias digitais policiais (SIDPo) representa uma oportunidade institucional para inovar, validar e reconhecer as competências e habilidades do profissional policial em ecossistemas digitais, ao facilitar a interação digital com a comunidade e o Estado em tempo real, especialmente naqueles eventos em que os avanços institucionais são desconhecidos devido à rigidez das comunicações oficiais.

Palavras-chave: ecossistema digital, inovação, insígnia, polícia, sistema

El uso de insignias para denotar identificación y reconocimiento dentro de una comunidad u organización es una práctica antigua. Los ejércitos romanos las utilizaban en estandartes para identificar a sus cohortes y legiones. Más tarde, en el siglo vi, las insignias iban grabadas sobre los escudos individuales, lo cual facilitaba el comando y control, así como el manejo táctico de las unidades en el campo de batalla (Berger, 1981; Webster, 1998).

En Europa medieval las insignias eran utilizadas como distintivos bordados en la vestimenta o acuñadas en distintas piezas metálicas para identificar a los individuos o familias. Hacia el siglo xiv, las insignias se heredaban y gozaban de amplia circulación (Cammann, 1944; Mayer, 1933). Durante la Segunda Guerra Mundial, los soldados eran reconocidos con insignias por su valentía y buena conducta (McAfee, 2015).

Comúnmente en organizaciones jerárquicas, las insignias no solo se utilizan para identificar a sus integrantes, sino también para demarcar rangos de autoridad. Tanto en órdenes religiosas como en organizaciones militares y policiales, la facultad de ejercer poder sobre otros individuos dentro de estas organizaciones está relacionada con el grado o el rango, el cual se marca claramente en los códigos que rigen el porte de uniforme (Sosis, 2006). En el caso de los cuerpos de policía, el uniforme 
y sus insignias representan el uso personificado del poder legítimo del Estado (Roach-Higgins \& Eicher, 1992).

En el ámbito educativo las insignias suelen utilizarse para identificar a aquellos que pueden ser confiados como colegatarios. En eventos deportivos, estas designan a quienes son del propio bando o son rivales, con lo cual facilitan el establecimiento de solidaridades y alianzas (Synott \& Symes, 1995).

En el ámbito digital, entre las primeras insignias que se popularizaron se registra la campaña "Blue Ribbon para la libertad de expresión en línea", promovida a mediados de la década de 1990 por la Electronic Frontier Foundation (EFF) como respuesta a la aprobación de una ley regulatoria de actividades en internet por el Congreso de los Estados Unidos. La campaña alentaba a los propietarios de sitios web a publicar un ícono digital con una cinta azul, que vinculaba mediante hipertexto al sitio web de la EFF, como distintivo para expresar solidaridad con el manifiesto de defensa de la libre expresión en línea. La insignia digital fue ampliamente adoptada, con su respectivo hipertexto, y como resultado el sitio EFF se convirtió en uno de los sitios más vinculados en la web (Klienberg, 1999).

Este tipo de insignias digitales cumple una finalidad similar a la de un botón, como los que comúnmente se usan para apoyar causas o candidatos, aunque tienen la funcionalidad adicional de dar contenido al perfil en línea de los usuarios que las exhiben y facilitan su vinculación con otros usuarios que apoyan la misma causa u otros intereses similares. Igualmente, se encontró una funcionalidad parecida en muchas insignias utilizadas en MySpace, lo que les permitía a los usuarios identificar su relación con una causa (Gueorguieva, 2008) o con una marca (Li, 2007).

De esta manera, las insignias digitales se han convertido en un vehículo importante para formar comunidades virtuales en redes sociales. A partir de entonces, plataformas como Mozilla Open Badge Framework y Khan Academy representan iniciativas que utilizan e impulsan las insignias digitales aplicadas a distintas finalidades (Ahn et al., 2014).

Muchas insignias digitales distinguen personas que han realizado determinadas experiencias, como, por ejemplo, el peregrinaje a un sitio sagrado o socialmente reconocido. Algunas de estas son certificadas por pares, o al menos suscitan sus comentarios. Varias otorgan logros alcanzados que requieren de sacrificios significativos de tiempo o energía, por lo cual cuentan con el respaldo de un sistema independiente y creíble de evaluación formal que las certifica, mientras que otras denotan autoridad, habilidad, experiencia e identidad. Muchas de ellas abarcan una colección compleja de todos estos atributos. En algunos casos, las insignias digitales combinan varias funciones complementarias; sin embargo, en ciertas situaciones, dichas combinaciones pueden resultar confusas y ser fuente de descrédito social para sus portadores (Halavais, 2012). Cuando las mismas insignias expresan, simultáneamente, tanto la motivación proveniente del reconocimiento horizontal de pares como la que deriva de observar las conductas esperadas por la autoridad jerárquica, su otorgamiento puede generar una disfunción dentro de la comunidad u organización, ya que la insignia se hace ambigua y pierde valor simbólico, tanto dentro como fuera de los contextos inmediatos en los que se concede (Robinson et al., 2012).

\section{Las insignias en la Policía Nacional de Colombia}

Doctrinalmente, en la Policía Nacional de Colombia, el reglamento de uniformes, insignias, condecoraciones y distintivos para su personal (Policía Nacional, 2009) dispone de las insignias y distintivos físicos con el objetivo de reconocer los comportamientos de los uniformados en su labor diaria, tendiente a garantizar la integridad de los ciudadanos, sus bienes y la convivencia en armonía, además de exaltar sus virtudes, méritos, habilidades o servicios. Sin embargo, en la actualidad la institución policial carece de un sistema de identificación y reconocimiento en entornos virtuales y digitales, que facilite la retroalimentación, motivación y acreditación y optimice su gestión de la convivencia y seguridad ciudadana.

El presente proyecto explora las condiciones de viabilidad para desarrollar e implementar un Sistema de Insignias Digitales Policiales (SIDPo) en la institución, como herramienta para motivar, retroalimentar, identificar, reconocer y acreditar las competencias, habilidades y aprendizajes del personal vinculado, a fin de contribuir a la mejora continua e innovación policial. Para ello se realiza un análisis de narrativas explicativas, desde la experiencia de los participantes que pertenecen a la institución en los ámbitos operativo y administrativo, permitiendo distinguir su conocimiento, percepción y construcción relacionados con los diferentes componentes necesarios para el diseño e implementación de un SIDPo, contribuyendo así al desarrollo personal y profesional de sus integrantes, en concordancia con la política de gestión del talento humano y cultura institucional. 
En principio, la propuesta se enmarca en la Resolución 01087 de 2019, por la cual se implementan las competencias genéricas en la Policía Nacional, con base en la siguiente definición:

Comportamientos comunes requeridos por la Policía Nacional para el cumplimiento de su misión y asegurar la efectividad en el servicio policial; son el fundamento para el éxito de la Institución y aportan atributos diferenciadores con cualquier otra organización del Estado.

Las competencias genéricas se enmarcan en cuatro dimensiones fundamentales: el ser (rasgos de personalidad), el saber (conocimientos), el saber hacer (habilidades) y el saber estar (interacciones en entornos). A ellas se asocian comportamientos exitosos observados en cada integrante, que contribuyen al cumplimiento efectivo de las funciones asignadas en el desempeño de sus respectivos cargos, y deben reflejarse en el grado en que se es competente para dar los resultados esperados a la luz de las dimensiones señaladas. Las competencias genéricas se definen a continuación en la tabla 1 :

Tabla 1

Competencias genéricas de la Policía Nacional

\begin{tabular}{|c|c|}
\hline Competencias & Definición \\
\hline Desarrollar relaciones & $\begin{array}{l}\text { Interactuar consigo mismo, con la insti- } \\
\text { tución y la comunidad de una manera } \\
\text { ejemplarizante y transformadora. }\end{array}$ \\
\hline Ser policía & $\begin{array}{l}\text { Reconocer sus propias capacidades y } \\
\text { habilidades, con el fin de alcanzar una } \\
\text { mejora continua policial, bajo parámet- } \\
\text { ros de ética y transparencia institucional. }\end{array}$ \\
\hline Servir con pasión & $\begin{array}{l}\text { Evidenciar comportamientos que de- } \\
\text { muestran actitudes de servicio e interés } \\
\text { por satisfacer las necesidades de la co- } \\
\text { munidad, con el fin de fortalecer la credi- } \\
\text { bilidad y confianza hacia la institución. }\end{array}$ \\
\hline Aprender para aportar & $\begin{array}{l}\text { Aplicar y desarrollar de manera continua } \\
\text { conocimientos, obteniendo rendimientos } \\
\text { evaluables que optimicen el desempeño } \\
\text { laboral. }\end{array}$ \\
\hline Actuar con efectividad & $\begin{array}{l}\text { Responder de manera efectiva a los } \\
\text { nuevos contextos que se presentan, man- } \\
\text { teniendo la calidad en la prestación del } \\
\text { servicio de acuerdo con los requerimien- } \\
\text { tos de la ciudadanía y la institución. }\end{array}$ \\
\hline
\end{tabular}

Nota. Policía Nacional (2019).

Mediante la referida resolución, la Policía Nacional adopta estas competencias como insumo fundamental de optimización en los procesos de selección e incorporación, formación, ubicación laboral, evaluación del desempeño, capacitación, inducción y reinducción, estímulos e incentivos y clima institucional. Les corresponde a la Dirección de Talento Humano, Dirección Nacional de Escuelas y Dirección de Incorporación liderar los procesos de divulgación, capacitación e implementación del Modelo de Gestión del Talento Humano y Cultura Institucional (MGTHCl), para la aplicación de las competencias genéricas en el personal uniformado de la Policía Nacional.

El problema de investigación radica en establecer si las competencias genéricas presentadas en la resolución citada representan un campo idóneo para desarrollar un proyecto piloto de diseño y aplicación de un SIDPo como apoyo a la gestión del desarrollo personal y profesional de sus integrantes. De ser así, el presente estudio también busca identificar qué papel cumplirían las insignias digitales para ser funcionales a las necesidades de evaluación, desempeño, ubicación laboral y otorgamiento de estímulos e incentivos en la gestión del talento humano dentro de la institución. Asimismo, sistematizar los aspectos prácticos que sería necesario resolver para esta implementación, respondiendo a la pregunta: ¿cuáles son las condiciones de viabilidad para implementar un SIDPo como herramienta para identificar, motivar, reconocer y medir las competencias genéricas en la gestión del desarrollo personal y profesional dentro de la Policía Nacional de Colombia?

\section{Consideraciones teóricas}

\section{Insignias digitales}

Las insignias están destinadas a caracterizar a los integrantes de una comunidad u organización específica, que comparten sus valores y expectativas; el valor de estas como distintivo visual que comunica significado exige que la función que se les asigna sea comprendida sin ambigüedades y respetada por la comunidad epistémica en la que tiene origen. Las mismas fomentan la participación de los interesados y permiten identificarse estrechamente con alguna experiencia de aprendizaje, para "aprender a ser" en lugar de "aprender a hacer" (Brown \& Adler, 2008).

Las insignias deben servir a la comunidad local, pero a menudo se espera que tengan una huella más amplia, aunque, en esas circunstancias, es difícil predecir cómo podrían interpretarlas aquellos fuera de la comunidad. Por ejemplo, puede ser que alguien no reconozca el significado de las insignias que lleva un policía de otro país, pero al menos la insignia que ostenta proporciona alguna 
indicación sobre su portador en el contexto de la organización policial a la que pertenece. Una insignia ampliamente reconocida construye capital simbólico dentro y fuera de una comunidad (Ahn et al., 2014).

En ese sentido, para el portador, una insignia reconocida dentro y fuera de su organización constituye un capital simbólico valioso. A raíz de la alta movilidad y flexibilidad que exige el mundo del siglo xxi, a menudo se espera que las insignias generen un impacto mayor que el mero reconocimiento físico por parte de su organización. Esta circunstancia dificulta predecir cómo las interpretarán aquellos fuera de la organización o comunidad de origen, en tanto las mismas no suelen ser aceptadas universalmente por otras comunidades $\mathrm{u}$ organizaciones, al carecer de información confiable sobre el rigor establecido para otorgarlas a sus portadores (Cheng et al., 2019; Ellis et al., 2016; Halavais, 2012; Shields \& Chugh, 2016).

Es así como las insignias digitales representan una manera de comunicar mediante símbolos empleando la tecnología, la cual se encarga de proporcionar el medio e infraestructura para su materialización. De este modo, el papel de las insignias en las relaciones humanas y del ecosistema digital en el que desenvuelven necesita explicaciones teóricas.

El presente estudio exploratorio estará orientado por dos teorías: la primera de ellas es la teoría de los signos (Peirce, 1999/1894), para comprender el empleo de los símbolos como una condición necesaria de la comunicación entre los individuos y de la conceptualización como parte esencial en la interacción humana con su entorno.

En griego, un reloj de fuego es un "símbolo", esto es, una señal acordada; un estandarte o una bandera es un "símbolo", una contraseña es un "símbolo", una insignia es un "símbolo"; el credo de una iglesia se llama símbolo, porque sirve como insignia o lema; una entrada de teatro se llama "símbolo"; todo ticket o cheque que le da a uno derecho a recibir algo es un "símbolo". Además, toda expresión de sentimiento se Ilamó un "símbolo". Tales fueron los principales significados de la palabra en el lenguaje original. (Peirce, 1999/1894, apartado 6)

En segundo lugar, la teoría crítica de la tecnología facilita el entendimiento respecto al valor e importancia de la interacción de una insignia en ecosistemas digitales y la manera como su alcance tecnológico refuta la visión instrumentalista de la tecnología como neutral y sin valor, así como la afirmación sustantiva de que las tecnologías son autónomas y están diseñadas para servir al propósito de su propietario (Feenberg, 2005).

\section{Competencias genéricas de la Policía Nacional}

Las competencias genéricas son herramientas para la gestión del talento humano de la Policía Nacional, orientadas a asegurar desempeños exitosos, comportamientos destacados y mejoramiento continuo del servicio a nivel nacional. Proporcionan el insumo fundamental para dar identidad a la profesión policial por parte de las unidades que conforman el proceso de direccionamiento del talento humano.

Se definen como comportamientos comunes requeridos por la Policía Nacional para el cumplimiento de su misión y para asegurar la efectividad en el servicio policial. Son el fundamento para el éxito de la institución, aportan los atributos diferenciadores con las demás organizaciones del Estado colombiano y reflejan el grado en que se es competente para dar los resultados esperados, caracterizando los elementos específicos de un desempeño destacado a la luz de las dimensiones del ser, saber, saber hacer y saber estar.

Las competencias genéricas y los comportamientos asociados adoptados mediante la Resolución 1087 de 2019 reflejan el sello de la cultura institucional y constituyen insumo fundamental de optimización en los procesos de selección e incorporación, formación, ubicación laboral, evaluación del desempeño, capacitación, inducción, reinducción, estímulos e incentivo y clima institucional.

\section{Sistema de insignias digitales (SID)}

Un SID se conceptualiza como una herramienta de identificación y reconocimiento que se soporta en metadatos a los que se puede acceder en línea, con potencial para desarrollar tanto motivación intrínseca como extrínseca. Permite reconocer y certificar logros de distinta índole obtenidos por integrantes de una organización o comunidad (Ahn et al., 2014; Foster, 2013; Kim, 2015).

La documentación de actividades y logros vía web se constituirá, cada vez más, en parte central de la comunicación humana cotidiana, para establecer la confiabilidad y reputación de cada individuo en los nuevos contextos en los cuales interactuarán. Actualmente, millones de usuarios en todo el mundo utilizan Flickr, Facebook, Twitter y LinkedIn, entre otras redes sociales, como sus principales fuentes de información e interacción diaria. Además de las características personales idiosincrásicas, la información recabada en la web incluye tanto los patrones de 
actividad en línea como data temporal y espacial sobre las actividades y logros alcanzados por cada individuo, incluso fuera de línea (Tumpa et al., 2020).

Cuando se diseñan e implementan de manera coherente en una organización, las insignias digitales permiten la conexión y el reforzamiento del compromiso, vocación y motivación, a partir de las identidades y valores comunes que comparten sus integrantes, así como su reconocimiento por parte de terceros.

Factores de viabilidad para un Sistema de Insignias Digitales (SID)

Rogers (2003) identifica cinco características específicas de las innovaciones (ventaja relativa, compatibilidad, complejidad, probabilidad y observabilidad), que se asocian a tasas variables de su adopción. Tal marco conceptual permite determinar los factores que impiden o facilitan la implementación exitosa de un SID.

Uno de los factores que puede facilitar la difusión y adopción de insignias digitales es la compatibilidad del programa con los valores y las necesidades de la comunidad u organización que las otorga (Carey, 2017). Si las insignias pretenden expresar autoridad, deben ser coherentes con la forma en que abordan las opciones éticas dentro de las estructuras jerárquicas a las que se refieren.

Si se conciben principalmente como forma de autoidentificación, también deben estar expresamente enmarcadas en un conjunto de valores que guían la manera como se evalúan, se otorgan y reflejan el uso y sentido que se les asigna. Es vital reconocer que cualquier sistema de insignias digitales conlleva un cúmulo de expectativas éticas, y que las que se desempeñan mejor se basan en expectativas que son consistentes, cohesivas y adecuadas a su contexto inmediato (Foster, 2013).

Otro factor facilitador para la difusión de un SID es la observabilidad del valor de las insignias, tanto interna como externamente, y su ventaja relativa para comunicar a las partes interesadas un propósito o función clara (Carey, 2017).

Dado que las insignias están destinadas a ser un atajo visual, es importante que se mantengan estables y reconocibles. Una de las funciones principales de las insignias y los sistemas de gestión que las respaldan es construir comunidad y generar confianza, por lo cual es conveniente que la exhibición visual de la insignia digital permanezca estable. Además, para evitar que se conviertan en un símbolo vacío, las insignias digitales deben incorporarse a sistemas de información y gobernanza bien comprendidos por las partes interesadas, en los que repose toda la información y los metadatos que respaldan cada insignia, incluyendo título, descripción, criterio de asignación, URL de la imagen, emisor, fecha de emisión, beneficiario, URL de la evidencia, fecha de caducidad, etiquetas, entre otros. Por lo tanto, es importante poder confiar en los mecanismos de aplicación que controlan quién puede emitir una insignia digital y en qué condiciones. Si bien la transparencia del proceso de credencial depende en gran medida del contexto de uso, es importante que el proceso de evaluación y emisión permanezca abierto y documentado.

En términos más generales, lo ideal es que la insignia no sustituya a otras formas de evaluar o comunicar valor. Particularmente en las comunidades participativas, es importante que las insignias cuenten con un respaldo de metadatos amplios y de fácil acceso.

Entre los factores que pueden representar barreras para la adopción exitosa de un SID se encuentran la falta de comprensión del propósito y valor de las insignias digitales, así como los obstáculos para su reconocimiento y validez, más allá de la organización que las otorga (Carey, 2017). El valor y respeto por una insignia suelen estar determinados por la cantidad de esfuerzo realizado para recibirla. Al diseñar un sistema de insignias destinado a motivar a los participantes, es clave recordar que aquellas que siguen siendo las más respetadas y duraderas son las que requieren una alta dosis de sacrificio. Las insignias obtenidas en contextos más jerárquicos a menudo implican un sacrificio significativo por parte del portador, a veces, el máximo sacrificio (Halavais, 2012).

Por lo anterior, un aspecto importante para cualquier insignia es su capacidad para actuar como foco de múltiples comunidades. Ello requiere que un SID disponga de capacidades para interactuar con otras plataformas con funcionalidad para agregar insignias, lo cual le permite al usuario recopilar y agregar logros de diferentes ámbitos organizacionales en la misma plataforma. Sitios como Score.ly intentan compilar las insignias digitales de sus usuarios de múltiples fuentes, y Mozilla dispone de una infraestructura abierta para compartir insignias. Dichos esfuerzos tienen como objetivo aumentar la transparencia de estas por medio de los límites contextuales, para poder implementarlas como marcadores de identidad en múltiples ubicaciones (Medler, 2009). 
Un factor adicional que puede entorpecer la adopción de un SID es que su gestión Ilegue a representar una carga de trabajo administrativo excesiva dentro de la organización en cuanto a la promoción, diseño, desarrollo, divulgación e implementación del sistema y de su respectivo programa de respaldo. Una demanda particularmente fuerte se asocia con la evaluación de los requisitos establecidos por los programas, sobre todo cuando los estándares de exigencia son rigurosos.

Naturalmente, esto trae consigo beneficios instruccionales, gracias a los programas de insignias digitales que refuerzan el compromiso de los funcionarios a cargo de su gestión. Sin embargo, el desafío en relación con este factor consiste en asegurar que se mantengan altos niveles de motivación en todas las dependencias que asumen nuevas cargas de trabajo administrativo como consecuencia de la entrada en funcionamiento de este sistema (Carey, 2017).

En breve sentido, el proceso de diseño, implementación y obtención de una insignia puede concebirse como una receta: si un ingrediente es incorrecto, el plato no funcionará. Por eso es fundamental comprender que el proceso de creación de insignias y su aplicación dependerá de que la institución tome la decisión de establecer un SIDPo con los recursos necesarios, otorgándole prioridad frente a otros proyectos que en la actualidad compiten por dichos recursos humanos, técnicos y financieros.

\section{Consideraciones normativas}

Una búsqueda bibliométrica en bases de datos con auditoría de pares como Scopus y WoS, desde el año 2015 a la fecha, permitió confirmar la ausencia de una normatividad en países y cuerpos de policía de la región que regule el empleo de insignias digitales.

Por otro lado, se acató el artículo 2 de la Ley 1266 de 2008, como normativa de protección de datos personales, para preservar la confidencialidad de la información suministrada por parte de los integrantes de la institución que participaron voluntariamente en el presente estudio.

\section{Método}

El presente estudio se basó en un enfoque cualitativo, que permita identificar cómo se entienden y construyen los conceptos relacionados con los componentes necesarios para el diseño e implementación de un SIDPo, con el fin de apoyar el cumplimiento de las políticas de gestión del talento humano y cultura institucional en materia de desarrollo personal y profesional de sus integrantes.

Según Gibbs (2012), "la investigación cualitativa pretende acercarse al mundo de 'ahí fuera' (no en entornos de investigación especializada como los laboratorios) y entender, describir y algunas veces explicar fenómenos sociales 'desde el interior'" (pp. 13-14).

De acuerdo con Vasilachis (2007), "este componente del diseño también adoptará características diversas de acuerdo con la tradición de investigación cualitativa elegida" (p. 86).

Como una primera aproximación, se realizó una profundización exploratoria. Con ese propósito, la investigación se centró en indagar a personas expertas y actualmente vinculadas a las dependencias que desempeñan un papel estratégico en el desarrollo del MGTHCI de la entidad, preguntándoles sobre sus experiencias, percepciones, creencias, expectativas y motivaciones en relación con su implementación práctica. Específicamente, se sondeó su parecer acerca de la conveniencia de utilizar una innovación como el SID para apoyar la aplicación e implementación de las competencias genéricas establecidas en la Resolución 1087 de 2019 dentro de los procesos de divulgación, capacitación e implementación del MGTHCl en los cuales intervienen desde sus respectivas dependencias, y en relación con la idea de introducir insignias digitales como un elemento de apoyo a la evaluación de desempeño, la ubicación laboral y el reconocimiento de estímulos e incentivos dentro de la institución.

Como lo señala Ortiz (2015), la metodología interpretativa-comprensiva se orienta a estudiar las acciones de los sujetos en determinados contextos de sus prácticas cotidianas y sus reflexiones sobre estas. En el desarrollo de este método, los investigadores se centraron en la descripción y comprensión de lo que es singular y particular a los sujetos observados, para identificar y validar aqueIlos elementos susceptibles de generalizarse.

Para ello se utilizó la técnica de los grupos focales, la cual consiste en manejar la comunicación entre investigadores y participantes en un espacio de interacción segura y constructiva que permita captar el sentir, pensar y vivir de un grupo representativo de individuos, para obtener plurales y variadas narrativas explicativas acerca de las experiencias, actitudes, creencias, percepciones, expectativas 
y motivaciones del grupo. Por otra parte: "El uso de esta técnica beneficia a los investigadores, pues permite el ejercicio de una postura crítica y dialéctica, al promover el inicio de una discusión abierta sobre temas específicos y a veces propiciar la construcción y deconstrucción de conceptos" (Silva et. al., 2013).

La técnica se cimienta en la premisa de que el conocimiento es una producción humana y no tiene una correspondencia lineal con la realidad. En el grupo focal, el investigador construye conocimiento confrontándolo con los elementos aportados por los participantes del grupo, para luego validar el resultado con fuentes externas independientes. Es un proceso dialógico que busca comprender cómo los sujetos encuentran y dan sentido a sus experiencias, percepciones, creencias y temores, y cuáles son las diferentes condiciones objetivas que afectan los procesos de producción de sentido y comprensión de la realidad en que se encuentran inmersos (González, 2007; Kitzinger, 1995).

\section{Prueba piloto}

En la planificación del trabajo se resolvió, en principio, adelantar una prueba piloto con doce uniformados adscritos a la Metropolitana de Policía de Cartagena (MECAR), con diferentes grados, cargos y funciones, con el fin de realizar una aproximación respecto a su percepción y conocimiento en cuanto a insignias digitales. Dicha población fue escogida teniendo en cuenta la facilidad de acceso a ellos por parte de los investigadores.

Siguiendo a Ortiz (2015), en la fase preparatoria del grupo focal se realizó una presentación con diapositivas, con el fin de aclarar los propósitos del ejercicio y establecer las reglas del juego, y se plantearon tres preguntas, relacionadas con cada uno de los objetivos específicos establecidos en la descripción del problema de la presente investigación. Las preguntas que se utilizaron para animar el trabajo de grupo fueron las siguientes:

- ¿Cómo se aplican las competencias genéricas en los procesos de divulgación, capacitación e implementación del MGTHCI?

- ¿Qué papel juega el reconocimiento de competencias genéricas en la evaluación del desempeño, la ubicación laboral y el reconocimiento de estímulos e incentivos?

- ¿Qué aspectos prácticos habría que resolver para la implementación de un SID con el fin de fortalecer y realizar el seguimiento de las competencias genéricas en el personal uniformado de la Policía Nacional?
El instrumento se validó durante la prueba piloto con asesoramiento de un experto metodológico. En el desarrollo de esta actividad se registraron los discursos generados y se insertaron en la matriz que se incluye en el anexo 1. Dicha información se sistematizó y se analizó mediante el programa NVivo versión 11.4.0.

También se elaboraron los instrumentos para el registro y clasificación de la información generada en la conversación (anexo 1). Luego se hizo una socialización entre los investigadores de la presentación y los instrumentos, al igual que se fijaron los parámetros para el registro y clasificación de las informaciones aportadas por los investigadores en el papel de observadores. Con esto se buscó presentar los resultados del grupo focal de manera clara, lógica, racional y con coherencia sistémica, como insumo que permita formular las condiciones de viabilidad necesarias para implementar el sistema de insignias digitales proyectado.

Finalmente, en el grupo focal con los uniformados de la Metropolitana de Policía Cartagena (MECAR) se puso en evidencia la poca apropiación, conocimiento y experiencia sobre el tema, lo que generó la necesidad de abarcar otro grupo de población para poder, de manera más objetiva, dar respuesta a la pregunta problema de esta investigación. Algunos comentarios y afirmaciones de los primeros participantes confirman lo antes expuesto:

- "Las insignias digitales es lo mismo que las condecoraciones, pero en internet".

- "Eso sirve solamente para los que tienen teléfonos inteligentes o buenos teléfonos".

- "Primera vez que escucho sobre insignias digitales".

- "Las insignias digitales es solo para los oficiales o los que tienen vara".

Grupo focal con expertos

Con base en estos hallazgos preliminares, se tomó la decisión de convocar a un equipo de seis expertos en innovación policial, con el fin de integrar un grupo focal en la ciudad de Bogotá D. C. que permitiera, con el diseño y ejecución de la guía de observación mencionada, encontrar respuestas a la pregunta problema de este estudio y a los objetivos planteados. Se convocaron funcionarios adscritos a seis dependencias de la institución que fueron identificadas como partes interesadas y cuya participación resulta clave, en vista del papel estratégico que desempeñan en el apoyo de estos procesos misionales para la innovación y mejoramiento continuo: Dirección de Ta-

\section{.}

3 
lento Humano (DITAH), Dirección Nacional de Escuelas (DINAE), Dirección de Incorporación (DINCO), Oficina de Telemática (OFITE), Oficina de Comunicaciones Estratégicas (COEST) y Oficina de Planeación (OFPLA).

El aporte buscado de estas dependencias en el grupo focal radica en su experiencia y visión, a fin de cumplir con el objetivo de explorar las condiciones de viabilidad para implementar el Sistema de Insignias Digitales Policiales (SIDPo) como herramienta para identificar, motivar, promover, retroalimentar y reconocer las competencias genéricas en la gestión del desarrollo personal y profesional dentro de la Policía Nacional de Colombia.

Este tipo de muestreo por propósito está basado en la asunción de que el conocimiento del investigador acerca de la muestra puede usarse para seleccionar los casos representativos para esta, de acuerdo con un criterio de importancia (Brill et al., 2006). Se definieron las dependencias que deberían participar en el grupo focal y los funcionarios que, por su misionalidad y experticia, podrían agregar valor al presente estudio.

La convocatoria se realizó con el apoyo de la Oficina de Planeación de la Dirección General, dentro de las iniciativas de innovación y mejoramiento continuo que se lideran desde esa dependencia. Con anterioridad se preparó una presentación del marco institucional en el cual se ubica el ejercicio del grupo focal, al igual que los elementos fundamentales acerca de lo que son las insignias digitales, los componentes necesarios para establecer un SID, su utilidad para apoyar la implementación de la política de gestión del talento humano y las dificultades previsibles para la aceptación de esta innovación organizacional por parte de los integrantes de la institución.

Dado que no debe esperarse coincidencia en la interpretación de las informaciones obtenidas del grupo focal, Galeano (2009) recomienda que estas se soporten sobre datos obtenidos de fuentes independientes para su validación externa.

Con el propósito de obtener diversas visiones del tema de estudio y aproximarse a una realidad más precisa, se optó por la triangulación como método de análisis e interpretación, basado en "muestras y conjuntos de datos dispares cronológica y geográficamente y datos obtenidos de entrevistas, observación y documentos" (Gibbs, 2012, p.153), e integrando las fuentes bibliográficas (temáticas, teóricas y metodológicas) con los testimonios clasificados. A partir de las fuentes disponibles, se cruzaron las interpretaciones consignadas por los investigadores para la construcción ordenada de una primera versión general del reporte, teniendo presente el planteamiento del problema de investigación. Una vez agrupados los temas, se procedió a sintetizar las ideas, ordenar metodológicamente los conceptos y construir la redacción final de los resultados de la investigación.

\section{Resultados}

De la información obtenida mediante las preguntas de los grupos focales surgió el siguiente análisis y sistematización de la información, producto de los discursos recopilados en relación con los objetivos establecidos para el presente estudio.

Dificultad para la implementación en las actuales competencias genéricas

En el año 2004, con el nacimiento del Modelo de Gestión del Talento Humano y Cultura Institucional (MGTHCl), se establecieron por primera vez nueve competencias genéricas, con el fin de describir el tipo de policía que requería la institución y las características que resultan necesarias para el cumplimiento de sus objetivos estratégicos.

En este sentido, en su implementación se busca establecer cuáles son los comportamientos asociados (predispositores) y los requeridos para formar y desarrollar dichas competencias, entendidos estos como las capacidades del uniformado para adquirir una habilidad, es decir, para que existan las condiciones comportamentales e individuales que garanticen que esa habilidad se va a requerir y se va a poder formar, lo cual se identificó como uno de los primeros factores para la modificación de las nuevas competencias genéricas, según uno de los expertos.

Por consiguiente, existe dificultad al momento de implementar las insignias digitales en las actuales competencias genéricas, toda vez que estas últimas no han desarrollado los predispositores y representarían un obstáculo al momento de incluir algunos metadatos en las insignias digitales, por la complejidad del acceso a la información respecto a su validación institucional.

Las competencias genéricas en la evaluación del desempeño, la ubicación laboral y el reconocimiento de estímulos e incentivos

Se evidenció que se podía evaluar a un funcionario durante su trayectoria para verificar si tenía o no una competencia específica y que, sin embargo, era imposible 
certificarla. Por otra parte, partir del año 2012 se establecieron los comités de revisión estratégica e innovación policial (CREIP):

[...] con el fin de generar propuestas estratégicas de implementación, cambio y mejora en la actividad de policía, para buscar un giro e impacto en términos de imagen, credibilidad y confianza, a través de un proceso de análisis, referenciación y observación concienzuda del entorno cambiante y de las exigencias de la sociedad frente al servicio de policía. (León, 2012, pp. 30-31)

Muchos de esos CREIP pusieron énfasis en la necesidad de ajustar y/o cambiar las nueve competencias genéricas existentes en la institución desde el año 2004, toda vez que las establecidas inicialmente no especificaban acertadamente lo que debería ser un profesional de policía, es decir, no certificaban las habilidades básicas de competencia laboral como, por ejemplo, mediación policial, manejo de armas de fuego, registro de personas, y todas aquellas que el uniformado requiere a diario para desarrollar su profesión. En otros términos, desde el establecimiento de las nueve competencias genéricas en la institución, y su posterior ajuste a cinco, no hubo consenso evidenciable respecto al papel que representa el reconocimiento en la evaluación del desempeño, la ubicación laboral y los estímulos e incentivos, situación que, según lo contemplado en la teoría de los signos, es necesaria para la comprensión de los símbolos e insignias como una condición comunicativa necesaria entre los individuos y su entorno.

Las conclusiones obtenidas en los CREIP constituyeron un hallazgo importante, ya que permitieron abrir una posibilidad para que las insignias digitales representen un aporte valioso en los procesos de identificación y reconocimiento dentro de la estrategia de formación establecida en el Sistema Táctico Básico Policial (SITAB), e influyan así en la motivación intrínseca de los uniformados, al despertar su iniciativa por obtener dichas insignias, previo cumplimiento de la ruta de aprendizaje establecida en el SIDPo, lo cual redundaría en un mejoramiento continuo del servicio de policía y, por ende, de la imagen institucional. En este sentido, uno de los expertos mencionó:

A mí me parece que sí tendría un mayor impacto emplear las insignias digitales en el Sistema Táctico Básico Policial, donde, si se trabaja en aquellos puntos en la institución que están generando una mala imagen, mal cuestionamiento por el manejo de armas letales y también de armas no letales, como la táser, por ejemplo, allí considero que sería el reto enfocarse en la parte de formar las habilidades básicas que requiere el uniformado a diario, y en donde la insignia digital podría iniciar institucionalmente; ya sería tiempo para normar, mientras se trabaja con las habilidades que se comentaban.

Sin embargo, otro de ellos afirmó:

Inclinarse por la implementación de las insignias digitales en las competencias genéricas no va a ser posible, por lo menos no en este momento, toda vez que se requieren fichas técnicas para certificar la habilidad; en cambio, si nos vamos por el Sistema Táctico Policial, tenemos unos requerimientos mínimos de la función policial con los que se puede generar la ficha de chequeo, y que ya están diseñados institucionalmente para la certificación de la competencia.

Aspectos prácticos en la implementación de un SID para fortalecer y realizar el seguimiento de las competencias genéricas en los uniformados de la Policía Nacional

Con respecto a este tema, en principio se requiere establecer las descripciones de las habilidades y competencias en diferentes fases, junto con los predispositores, en tanto son aquellas las que funcionan para los procesos de selección e incorporación policial.

Luego es necesario diseñar otra descripción, desde la fase de la educación para los desarrollos de la formación policial, y otra más desde la fase de la competencia laboral para el desarrollo personal y profesional de sus integrantes; se puede decir que se trata de las mismas competencias descritas por factores comportamentales en tres niveles diferentes. Según una de las expertas:

[En otras palabras,] es la descripción de las pruebas psicotécnicas para la medición de esos predispositores de habilidades que se iban a dar en alguna de las fases, y era una sola prueba midiendo en uno de los tres momentos diferentes cada una de esas habilidades.

Otro de los expertos afirmó:

Si le preguntamos a cualquier funcionario, no tiene ni la más remota idea de cuáles son las competencias genéricas ni cómo están descritas, porque, a pesar del esfuerzo institucional para realizar la mejor divulgación, la realidad es que no ha llegado todavía a interiorizarse de manera integral dentro de la institución.

Además, se debe tener en cuenta que esas competencias genéricas son ciento por ciento habilidades blandas, que por lo general no se certifican ni se estandarizan; realmente se certifica lo que se sabe hacer de manera tangible y medible, es decir, la idoneidad en la competencia 
laboral, si bien lo único que otorga la idoneidad es una certificación académica y lo que certifica como una habilidad laboral es el hacer, tanto como el saber hacer.

Asimismo, se identificó el poco interés o motivación por parte de los uniformados para recibir reentrenamiento, lo cual es necesario para la prestación de un servicio policial con efectividad. El hecho de que los policías no se sientan incentivados o motivados es una consecuencia de que culturalmente se dé más prioridad a que estén en la calle cumpliendo las horas de servicio, por lo que también se les debe dar tiempo para que reciban el entrenamiento adecuado. Este podría ser uno de los principales aspectos que explican por qué esos ejercicios de reentrenamiento se ven de esa manera, debido a que, según uno de los expertos:

Por cumplir, van a la capacitación, reciben la instrucción, diligencian la planilla de asistencia, y al final del día es como si no hubiesen aprendido nada; ni qué decir de la práctica constante y/o retroalimentación de las técnicas para la mediación policial, o para una persecución policial, y demás necesarias para el servicio, no se observa la práctica constante.

Un último hallazgo obtenido está relacionado con la incorporación del SITAB como uno de los retos del Plan Marco de Innovación Policial. En palabras de uno de los expertos:

En atención a los lineamientos del señor director general de la Policía y de las actividades del Pilar nro. 2: "Una policía que se transforma para servir mejor", la Oficina de Planeación, en coordinación con las direcciones y oficinas asesoras, diseñó el Plan Marco de Innovación Policial, el cual busca implementar la innovación mediante la planeación estratégica institucional en sus procesos y procedimientos, con el fin de dar el salto organizacional en materia de innovación y avanzar en el cumplimiento a los lineamientos para la función pública en la materia, así como en el desarrollo del Modelo Integrado de Planeación y Gestión.

En este sentido, uno de los retos de innovación de dicho plan marco está relacionado con la gamificación y la preparación mental, comunicativa y táctica de los uniformados, como factores integrados en el SITAB.

Lo anterior hace evidente que el diseño e implementación de un SID aportaría al reto de innovación establecido en el plan marco mencionado y solucionaría la necesidad institucional de mejorar los procesos de formación y re- conocimiento de sus uniformados, lo cual, conforme la teoría crítica de la tecnología, facilitaría el entendimiento respecto al valor e importancia de la insignia digital al momento de interactuar con la cultura policial.

Con base en estos hallazgos, surge la idea de reorientar la construcción de un Sistema de Insignias Digitales (SID) hacia las competencias requeridas para la preparación mental, comunicativa y táctica del uniformado, asociadas al SITAB.

Principales aspectos para desarrollar las SIDPo

En relación con lo anterior, la figura 1 representa las categorías y subcategorías emergentes para el desarrollo de las insignias digitales: la primera categoría está relacionada con la educación policial, que comprende los procesos de motivación, retroalimentación y acreditación del aprendizaje policial de sus integrantes, mientras que la segunda se enfoca en los procesos de identificación y reconocimiento de las competencias, habilidades, experiencias y logros de sus integrantes, como principal impulsor para su desarrollo personal y profesional.

Figura 1

Categorías y subcategorías de insignias digitales de la Policía

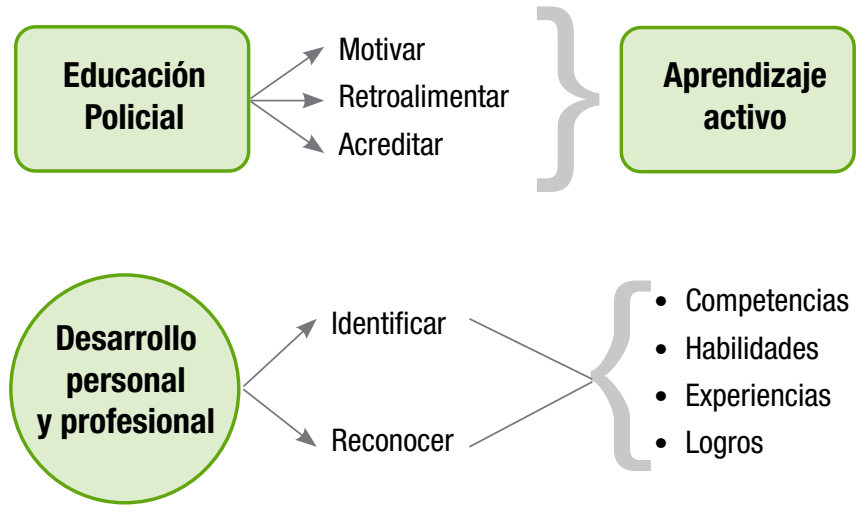

Por su parte, la nube de palabras de la figura 2, que surge como resultado de la discusión refleja los principales aspectos que se deben tener en cuenta para el diseño e implementación de un SIDPo, de modo que faciliten y fortalezcan la educación policial y promuevan el desarroIlo personal y profesional de sus integrantes.

En relación a los anteriores resultados podemos analizar y confirmar, desde un marco teórico, cómo las diferentes percepciones en la experiencia de los participantes reflejan la necesidad de implementar y diseñar estrategias como las insignias digitales en el ámbito policial, 
desde algunos de sus campos, como el talento humano, o en programas como el sistema táctico básico. Aquí se destacan ciertas categorías y subcategorías emergentes que demuestran que el ser humano se encuentra por naturaleza en un constante desarrollo cognitivo relacionado con la interacción en su entorno, lo que genera el afán de construir una identidad propia y el interés de pertenecer a una comunidad para cumplir sus necesidades básicas y crecer personal y profesionalmente, demostrando sus capacidades y habilidades frente a cualquier condición de tiempo y espacio a la que deba adaptarse, teniendo en cuenta que la sociedad es dinámica y evolutiva.

Figura 2

Nube de palabras de insignias digitales de la Policía

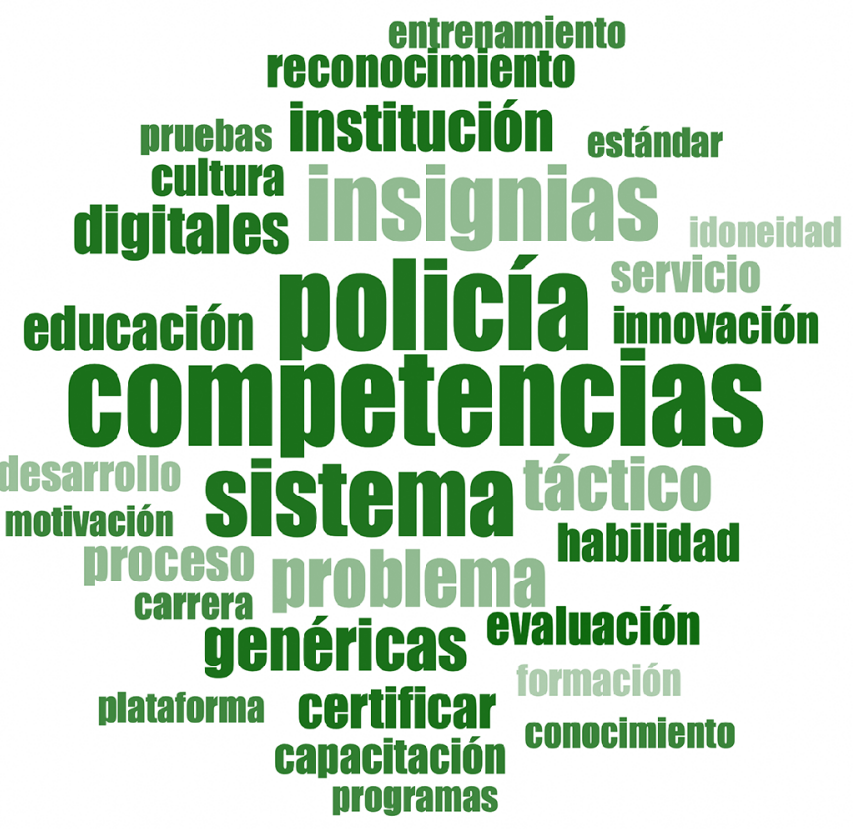

Es por ello que las insignias constituyen un símbolo que genera significado y valor en el individuo, como lenguaje de comunicación y representación hacia su comunidad, ya sea que se trate de conceptos, acciones o pensamientos que se atribuyen a sus experiencias personales y colectivas. Es decir que todo lo que sea interpretable se considera como un signo, y el hecho de que los individuos usen los signos para comunicarse es lo que los hace humanos, porque: "La palabra o signo que el hombre usa es el hombre mismo" (Peirce, citado en Salguero, 2001), en el entendido de que un símbolo se convierte en signo en el momento que se le genera un significado, tal y como se refleja en el Sistema de Insignias Digitales.
Integrar el contexto tecnológico desde las insignias digitales, como tecnología instrumentalizada, permite que el individuo (en este caso el funcionario y la institución) se relacione con los avances y dinámicas constantes en que se encuentra inmersa la sociedad, generando beneficios y oportunidades que faciliten y mejoren la calidad laboral y de vida, tanto personal como colectiva, desde el desarrollo de procesos y procedimientos en torno a los diferentes ámbitos socioculturales vinculados al emprendimiento e innovación, dirigiéndose a la motivación, el reconocimiento y los estímulos. La tecnología se ha convertido así en un poder social, creando la necesidad que las comunidades se organicen en torno a ella, mediante la construcción de diseños íntegros y unificados donde prevalezca el interés y las preocupaciones de que multiplique oportunidades y fomente la comunicación en las instituciones dependientes de ella. (Feenberg, 2005).

\section{Conclusiones}

Las insignias digitales representan una oportunidad institucional que permite y facilita la interacción digital con la comunidad y el Estado en tiempo real, especialmente en aquellos eventos en que los logros institucionales son desconocidos por la rigidez en las comunicaciones oficiales.

Pero validar las competencias y habilidades del profesional de policía en ecosistemas digitales requiere de un proceso de adaptación a la modernidad, en la cual las insignias digitales cumplen un papel facilitador, al ser interactivas y con unos metadatos que indican el logro y el esfuerzo realizado para poder alcanzarlo, sumando a ello su alto nivel de sociabilidad, que permite confirmar la competencia y la habilidad para saber emplearlas y desarrollarlas productiva y/o efectivamente.

Es necesario entonces el diseño de un proceso-ruta diferenciado para cada necesidad, como el evidenciado en el ejemplo de la figura 3, empleando escenarios de aprendizaje para la preparación mental, comunicativa y táctica del uniformado, que orienten el camino para la obtención de estas insignias a medida que se van alcanzando logros sobre la base de la cultura policial, la comunicación estratégica y la innovación. Para la implementación de este proceso-ruta se recomienda tener en cuenta los criterios de concientización, familiaridad, consideración y uso expuestos por Shields \& Chugh (2016); asimismo, se sugiere que se especifiquen los niveles, etapas o fases, objetivos y criterios que debe reunir el interesado en obtener la insignia digital, a fin de iden- 
tificar y reconocer los logros obtenidos en los aspectos de su preparación mental, comunicativa y táctica, los cuales se establecen en el SITAB como los "tres compo- nentes que se articulan para una regulación profesional en la atención de los procedimientos policiales" (Policía Nacional, 2018, p. 2).

Figura 3

Ejemplo proceso - ruta SIDPo

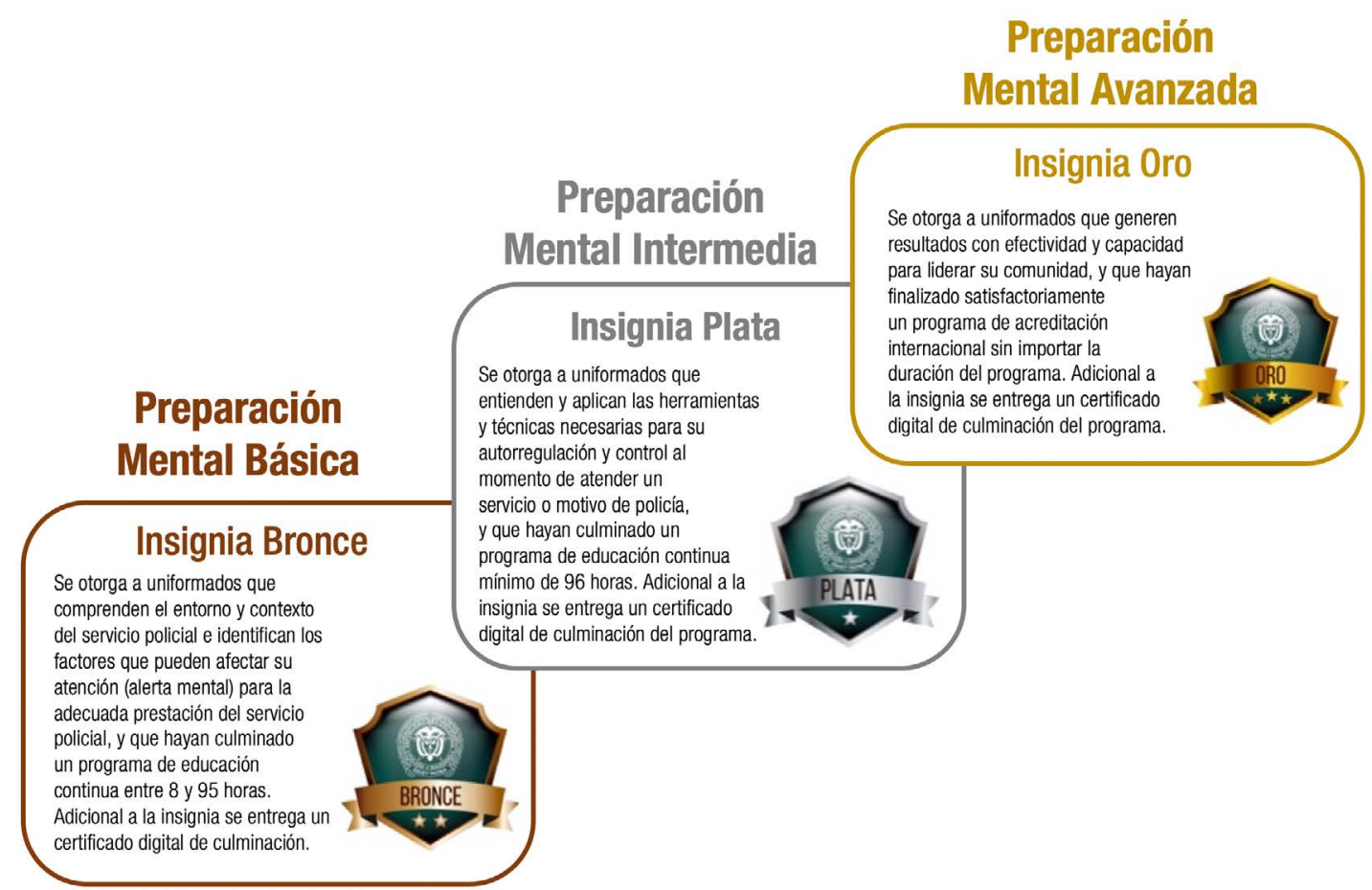

Las iniciativas y desarrollos prospectivos adelantados dentro de la institución, como los adelantados desde el año 2012 con el CREIP y con el más reciente Plan Marco de Innovación Policial, demuestran la constante búsqueda institucional por actualizarse y adelantarse a los cambios que la tecnología genera en las sociedades, con el propósito de mejorar la efectividad policial, teniendo en cuenta que en un futuro cercano la planta de personal de la Policía Nacional estará conformada en su totalidad por nativos digitales, que se espera interactúen en un mercado laboral inmerso en un ecosistema digital.

Lo anterior se suma al establecimiento de un proceso de aprendizaje formal y continuo, tanto para los instructores policiales como para los integrantes de la institución, que facilite la implementación de las insignias digitales en el Sistema Táctico Básico (SITAB), dado que estas solo se validan mientras se sigan desarrollando y mantengan su valor.

Entre las limitaciones a resolver están la creación de un inventario de insignias digitales para la preparación mental, comunicativa y táctica del uniformado, el diseño de las representaciones visuales y su incorporación e implementación en el SIDPo, y la digitalización de la experiencia (interacción) con el servicio de policía para su inclusión en los metadatos de las insignias. Estos últimos pueden incluir el logo de la unidad policial que otorga la insignia, el tipo de certificación, la competencia, habilidad o logro, el nombre del integrante, el nombre de la unidad que la emite, la fecha de emisión y expiración, la descripción del logro, competencia o habilidad alcanzada, los requisitos que debieron cumplirse para su otorgamiento y las evidencias.

Por otro lado, teniendo en cuenta que la planta de personal está conformada en su mayoría por uniformados en el grado de patrulleros y que el histórico de retiros de esta población viene en aumento desde el año 2010 hasta la fecha (figura 4), una posible línea futura de investigación se relaciona con los aportes que las insignias digitales pueden representar en los ejercicios de identificación, motivación y reconocimiento de los uniformados, y su incidencia en la fidelización y motivación para permanecer en la institución. 
Figura 4

Histórico de retiro de patrulleros por solicitud propia en 2010-2020 y proyección para 2024

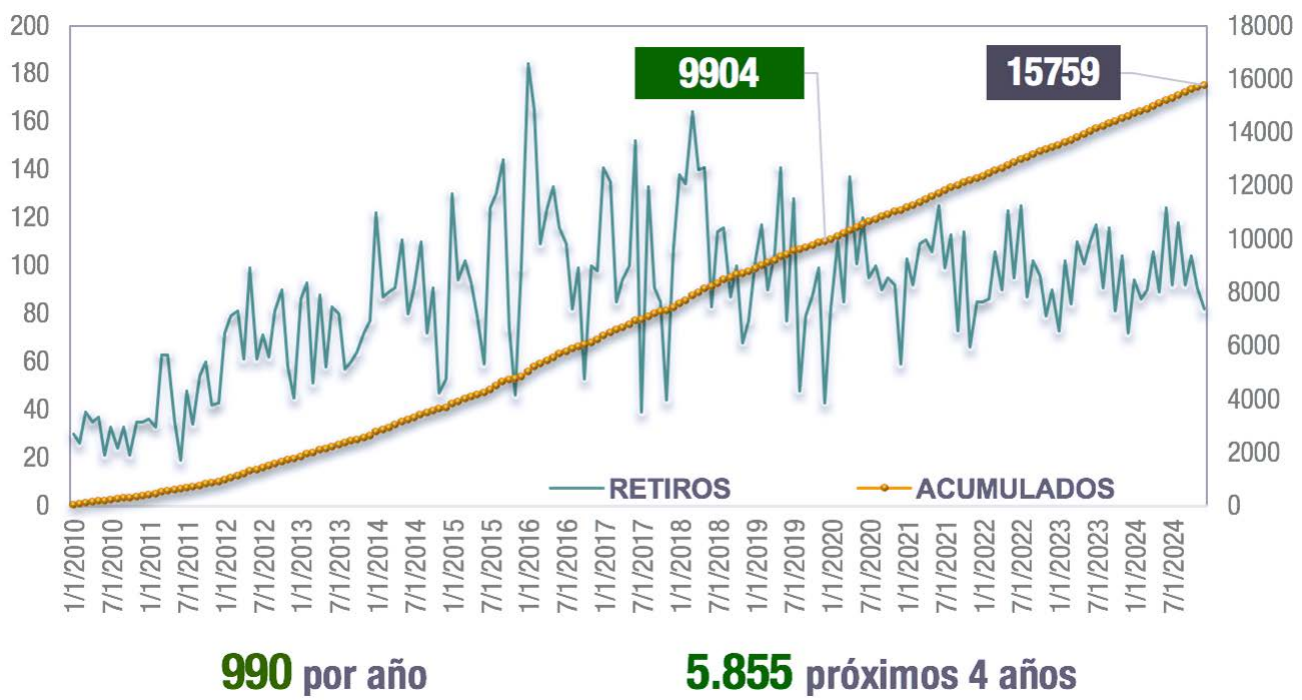

Nota: Dirección de Talento Humano - Oficina de Planeación (2020).

Finalmente, se considera importante establecer en un próximo estudio los aspectos relacionados con la implementación e impacto de las insignias digitales en el Sistema Táctico Básico Policial (SITAB), que permitan identificar, motivar, promover, retroalimentar y reconocer la calidad en la preparación mental, comunicativa y táctica del uniformado y su incidencia en la efectividad, mejora continua e innovación del servicio policial.

Sin embargo, desde los hallazgos presentados se deben tener en cuenta los posibles escenarios a futuro que se generarían en el momento de diseñar e implementar el SIDPo, tales como, por citar algunos, su incidencia en la cultura policial y en la manera de reconocer y exaltar logros y habilidades, en los procesos de formación e innovación policial, y en su entendimiento, aceptación e implementación por parte de las generaciones que no son nativos digitales, además de la capacidad de adaptación, respuesta y crecimiento (escalable y repetible) de las insignias digitales en todas las unidades policiales del país.

\section{Referencias}

Ahn, J., Pellicone, A. \& Butler, B. (2014). Open badges for education: What are the implications at the intersection of open systems and badging? Research in Learning Technology, 22. https://doi.org/10.3402/rlt. v22.23563
Berger, P. C. (1981). The Insignia of the Notitia Digitatum. Garland.

Brill, J. M., Bishop, M. J. \& Walker, A. E. (2006). The Competencies and Characteristics Required of an Effective Project Manager: A Web-Based Delphi Study. Education Tech Research and Development, 54, 115-140. https://doi.org/10.1007/s11423-006-8251-y

Brown, J. S. \& Adler, R. P. (2008). Minds on fire: open education, the long tail, and learning 2.0. Educause Review, 43(1), 16-32.

Cammann, S. (1944). The development of the Mandarin square. Harvard Journal of Asiatic Studies, 8(2), 71-130.

Carey, K. (2017). An Analysis of Factors that Impact Diffusion and Adoption of Digital Badges [doctoral dissertation]. ODU Digital Commons. Old Dominion University. https://digitalcommons.odu.edu/cgi/ viewcontent.cgi ?article $=1025 \&$ context=stemps_etds

Cheng, Z., Richardson, J. \& Newby, T. J. (2019). Using digital badges as goal-setting facilitators: A multiple case study. Journal of Computing in Higher Education, 32, 406-428. https://doi.org/10.1007/s12528-01909240-z

Ellis, L., Nunn, S. G. \& Avella, J. (2016). Digital Badges and Micro-credentials. Historical Overview, Motiva- 
tional Aspects, Issues, and Challenges. En D. Ifenthaler, N. Bellin-Mularski \& D. K. Mah (Eds.), Foundation of Digital Badges and Micro-Credentials. Demonstrating and Recognizing Knowledge and Competencies (pp. 3-21). Springer. https://doi.org/10.1007/978-3319-15425-1

Feenberg, A. (2005). Critical theory of technology. Tailoring Biotechnologies, 1(1), 47-64.

Foster, J. C. (2013). The promise of digital badges. Techniques, 88(8), 30-34.

Galeano, M. (2009). Estrategias de investigación social cualitativa. El giro en la mirada. Carreta Editores.

Gibbs, G. (2012). El análisis de datos cualitativos en investigación cualitativa. Morata.

González, R. (2007). Investigación cualitativa y subjetividad. Los procesos de construcción de la información. McGraw Hill.

Gueorguieva, V. (2008). Voters, MySpace, and YouTube: the impact of alternative communication channels on the 2006 election cycle and beyond. Social Science Computer Review, 26(3), 288-300.

Halavais, A. M. C. (2012). A Genealogy of Badges. Information, Communication \& Society, 15(3), 354-373. https://doi.org/10.1080/1369118X.2011.641992

Kim, J. (2015, 5 de enero). The value problem in digital badging. Inside Higher Ed. https://www.insidehighered.com/blogs/technology-and-learning/ value-problem-digital-badging

Kitzinger, J. (1995). Qualitative Research: Introducing Focus Group. BMJ, 311, 299-302. https://doi. org/10.1136/bmj.311.7000.299

Klienberg, J. A. (1999). Authoritative sources in a hyperlinked environment. Journal of the ACM, 45(5), 604-632.

León, J. (2012). Una institución a la vanguardia de la sociedad, Comité de Revisión Estratégica e Innovación Policial - CREIP. Revista Policía Nacional, 292, 30-31. https://www.policia.gov.co/file/36333/ download?token=UvxJ5-G_

Li, C. (2007). How Consumers Use Social Networks. Forrester Research.
Mayer, L. A. (1933). Saracenic Heraldry: A Survey. Oxford University Press.

McAfee, M. J. (2015). Service, war and veteran stripes: True badges of distinction. Military Images, 33(1), 49.

Medler, B. (2009). Generations of game analytics, achievements and high scores. Eludamos, 3(2), 177-194.

Ortiz, A. (2015). Enfoques y métodos de investigación de las ciencias humanas y sociales. Ediciones de la $\mathrm{U}$.

Peirce, C. S. (1999). ¿Qué es un signo? En El arte de razonar (Trad. U. Rivas). (Trabajo original publicado en 1894). https://www.unav.es/gep/Signo.html

Policía Nacional de Colombia (2009). Resolución 3372 de 26 de octubre. Por la cual se expide el Reglamento de uniformes, insignias, condecoraciones y distintivos para el personal de la Policía Nacional. Dirección General.

Policía Nacional de Colombia (2018). Guía práctica del sistema táctico básico policial. Código 3EC-GU-0001. Dirección Nacional de Escuelas - Policía Nacional de Colombia.

Policía Nacional de Colombia (2019). Resolución 01087 de 29 de marzo. Por la cual se implementan las competencias genéricas de la Policía Nacional. Dirección General.

Roach-Higgins, M. E. \& Eicher, J. B. (1992). Dress and identity. Clothing \& Textiles, 10(4), 1-8.

Robinson, L. J., Stevens. L. H., Threapleton, C. J. D., Vainiute, J., McAllister-Williams, R. H., \& Gallagher, P. (2012). Effects of intrinsic and extrinsic motivation on attention and memory. Acta Psychologica, 141(2), 243249. https://doi.org/10.1016/j.actpsy.2012.05.012

Rogers, E. M. (2003). Diffusion of innovations. Free Press.

Salguero, F. J. (2001). Teoría General de los Signos y del Significado. En A. Nepomuceno, J. F. Quesada \& F. J. Salguero (eds.), Información: Tratamiento y Representación (pp. 41-58). Servicio de Publicaciones. Universidad de Sevilla.

Shields, R. \& Chugh, R. (2016). ¿Digital badges - rewards for learning? Journal Education \& Information Technologies, 22, 1817-1824. https://doi.org/10.1007/ s10639-016-9521-x 
Silva, M. G., Fernandes, J. D., Rebouças, L. C., Rodrigues, G. R. S., Teixeira, G.A. \& Silva, R.M.O. (2013). Publicações que utilizaram o grupo focal como técnica de pesquisa: ¿o que elas nos ensinam? Ciência, Cuidado e Saúde, 12(2), 398-406.

Sosis, R. (2006). Religious behaviors, badges, and bans: signaling theory and the evolution of religion. En P. Macnamara (Ed.), Where God and Science Meet: Evolution, Genes, and the Religious Brain (vol. 1, pp. 6186). Praeger Publishers.

Synott, J. \& Symes, C. (1995). The genealogy of the school: an iconography of badges and mottoes. British Journal of Sociology of Education, 16(2), 139-152.
Tumpa, S., Kumar, P., Sultana, M., Hsu, G. S., Yadid-Pecht, O., Yanushkevich, S. \& Gavrilova, M. L. (2020). Social Behavioral Biometrics in Smart Societies. En M. Sarfraz (Ed.), Advancements in Computer Vision Applications in Intelligent Systems and Multimedia Technologies (pp. 1-24). IGl Global. https://doi:10.4018/978-17998-4444-0

Vasilachis, I. (Coord.) (2007). Estrategias de investigación cualitativa. Gedisa

Webster, G. (1998). The Roman Imperial Army of the First and Second Centuries A. D. University of Oklahoma Press. 